\title{
Composition chimique et dégradabilité enzymatique et in vitro d'espèces ligneuses arbustives utilisables par les ruminants dans les parcours extensifs de la Nouvelle-Calédonie. II. Equations de prédiction de la dégradabilité enzymatique et in vitro de la matière organique et des matières azotées
}

\author{
$\begin{array}{ll}\text { C. Corniaux }{ }^{1} \text { H. Guerin } 2 & \text { H. Steingass } \\ & \end{array}$
}

\section{Mots-clés}

Plante ligneuse - Valeur nutritive Analyse statistique - Composition chimique - Digestibilité - Matière organique - Matière azotée digestible Ruminant - Parcours - NouvelleCalédonie.

\section{Résumé}

En Nouvelle-Calédonie, comme dans de nombreux autres pays, il n'est pas envisageable à court terme de mettre en ouvre toutes les méthodes d'évaluation des fourrages. Par ailleurs, la valeur alimentaire des arbustes fourragers est relativement méconnue comparativement à celle des herbacées. Aussi cette étude établit-elle des équations de prédiction de quelques facteurs lićs à la valcur nutritive des ligneux tropicaux récoltés sur les zones de parcours néo-calédoniens. Il' s'agit, d'une part de critères déterminés par les méthodes enzymatiques : dégradabilité enzymatique de la matière organique (SMO) et des matières azotées dégradées par la pronase (MApro) ; et, d'autre part, par la méthode in vitro : dégradabilité in vitro de la matière organique (dMOgt) et des matières azotées (MAgt) par la méthode de production des gaz. Cette étude montre notamment la difficulté de déterminer ces équations à partir de critères chimiques simples ( $M \wedge T$, NDF, ADF) couramment utilisés pour les espèces herbacées. Leur teneur généralement élevée en lignines, la distribution de leurs matières azotées partiellement bloquées au niveau des parois indigestibles et la présence de tanins rendent nécessaire l'emploi de critères chimiques tels qu' $A D L, M A a d f$ et MAnadf (matières azotées liées ou non à I'ADF) dans le cas des arbres et arbustes fourragers. La précision des équations est néanmoins acceptable notamment pour la dMOgt et pour la famille des Fabacées. En revanche, les équations ne sont pas applicables pour MApro (sauf pour les Fabacées).

\section{INTRODUCTION}

Les ligneux fourragers des zones tropicales jouent un rôle important dans l'élevage des ruminants, notamment pendant la saison sèche. C'est pourquoi le choix des espèces à exploiter, qui repose souvent sur des critères agronomiques (productivité, fixation

1. CIRAD-Elevage, Port Laguerre, BP 186 Nouméa, Nouvelle Calédonie

2. CIRAD-EMVT, Campus international de Baillarguel, BP 5035, 34032 Montpellier, France

3. Institït für Tierernährung, Universität Hohenheim, Allemagne d'azote, limitation de l'érosion, résistance à la sécheresse), se fait également sur leur valeur alimentaire. Mais cette dernière est méconnue aujourd'hui comparativement à celles des espèces herbacées. En conséquence, il existe très peu d'équations de prédiction de critères synthétiques de la valeur énergétique et azolée des arbustes fourragers $(2,12)$.

En Nouvelle-Calédonie, comme dans la plupart des pays de la région Pacifique (Australie, Indonésie), la régression du Leucaena leucocephala, largement utilisé dans les systèmes d'alimentation des ruminants en saison sèche (20), a conduit les auteurs à compléter la gamme des espèces ligneuses à tester par des espèces lo- 
cales, identifiées soit comme étant appétées couramment ou accidentellement par le bétail domestique ou sauvage, soit appartenant aux familles de Légumineuses fixatrices d'azote. Les premiers travaux $(8,9,10)$ ont permis d'établir une typologie de 45 arbres ou arbustes répondant à ces critères et basée sur leur composition chimique et sur leurs digestibilités enzymatiques ou in vitro.

A partir des nombreuses analyses réalisées lors de ces travaux, l'étude présentée ici vise à proposer des équations de prédiction de différents critères liés à la valeur nutritive des arbustes fourragers. Elles faciliteront leur évaluation à partir de critères simples mesurés en laboratoire.

\section{MATERIEL ET METHODES}

\section{Les ligneux fourragers}

Quarante cinq échantillons d'organes d'espèces ligneuses arbustives ont été récoltés en juillet et en août 1993 (9). Ils étaient principalement constitués de feuilles et de tiges non chlorophylliennes et parfois de fleurs ou de fruits (10). La collecte a été effectuée dans le sud de la Nouvelle-Calédonie (Païta), sur la côte Ouest et la côte Est ainsi que sur les îles Loyauté. Les échantillons ont été placés à l'étuve en sachet papier pendant $72 \mathrm{~h}$ à $70^{\circ} \mathrm{C}$.

\section{Les mesures}

\section{Les laboratoires d'analyse}

Trois laboratoires ont analysé les échantillons ; le premier en Nouvelle-Calédonie (Laboratoire d'analyse des aliments du bétail, noté LAAB), le second en France (Laboratoire d'alimentation CIRAD-EMVT de Maisons-Alfort), le troisième en Allemagne (Institut für Tierernährung, Universität Hohenheim).

\section{Les analyses}

Les principales méthodes d'analyses chimiques sont celles classiquement appliquées aux aliments du bétail et décrites par l'AFNOR (1) ou le BIPEA (5). Les références des autres méthodes sont indiquées ci-dessous.

Les critères chimiques mesurés sont :

- matière sèche, notée MS (p. 100 de la matière fraîche)

- matières minérales, notées MM (p. $100 \mathrm{MS}$ )

- matières azotées totales, notées MAT (p. 100 MS)

- matières grasses, notées MG (p. 100 MS)

- cellulose brute, notée CB (p. 100 MS)

- neutral detergent tiber, noté NDF (p. $100 \mathrm{MS}$ ) (23)

- acid detergent fiber, noté ADF (p. $100 \mathrm{MS}$ ) (23)

- acid detergent lignin, noté ADL (p. $100 \mathrm{MS}$ ) (23)

- MAadf : matières azotées liées à l'ADF (p. 100 MS) (16). Par différence aux MAT, on obtient les matières azotées non liées à l'ADF, notées MAnadf

- les teneurs en tanins précipitants (p. $100 \mathrm{MS}$ ), notés tanpt (11)

- les teneurs en tanins condensés (p. $100 \mathrm{MS}$ ), notés tanc (19).

Les critères enzymatiques mesurés sont :

- la dégradabilité enzymatique de la matière organique par la pepsine cellulase, notée SMO (p. $100 \mathrm{MS}$ ) (4)

- la dégradabilité des matières azotées par la pronase après une heure d'incubation, notée DE1 (p. 100 MAT) (6). On obtient ainsi les teneurs en matières azotées dégradées par la pronase, notées MApro (p. 100 MS).

Les critères de dégradabilité in vitro mesurés sont :

- la production de gaz mesurée lors du gastest de Hohenheim à partir de laquelle est estimée la digestibilité de la matière organique, notée dMOgt (p. $100 \mathrm{MS}$ ) (17) ainsi que les matières azotées dégradées in vitro, notées MAgt.

L'ensemble de ces critères mesurés ou estimés seront testés pour déterminer les équations de prédiction dans l'analyse statistique.

\section{L'analyse statistique}

Il n'est pas toujours possible de mettre en cuvre toutes les méthodes d'évaluation de la valeur nutritive des fourrages. C'est le cas en Nouvelle-Calédonie, au moins à court terme, pour les méthodes in vitro de détermination de la dégradabilité de la matière organique et des matières azotées.

Aussi, à partir des critères chimiques seuls, des équations de régression ont d'abord été établies pour prédire des critères enzymatiques :

- SMO : facteur de la valeur énergétique des fourrages

- MApro : factcur de la valcur azotée des fourrages.

Puis, à partir des critères chimiques et enzymatiques, des équations de régression ont été établies pour prédire des critères in vitro:

- dMOgt : facteur de la valeur énergétique des fourrages

M $\Lambda \mathrm{gt}$ : facteur de la valeur azotée des fourrages.

Actuellement, ce sont ces critères qui expriment le mieux la valeur nutritive des arbustes fourragers $(2,12)$.

Les équations de régression, établies en stepwise sur SPSS (21), ont été calculées pour chacune de ces quatre variables sur :

- l'ensemble des ligneux fourragers (soit 45 espèces)

- les Légumineuses (soit 30 espèces)

- les Mimosacées (soit 18 espèces)

- les Fabacécs (soit 9 espèces)

- les non-Légumineuses (soit 15 espèces).

RESULTATS

\section{Prédiction de la SMO (tableau I)}

Les équations de prédiction de la dégradabilité enzymatique à la pepsine cellulase de la matière organique sont relativement fiables $\left(\mathrm{R}^{2}>=0,78 ; \mathrm{CVr}\right.$ de l'ordre de 10 p. 100$)$, notamment pour les Légumineuses. Il faut noter que, à l'instar des fourrages herbacés, le critère NDF n'est pas suffisant pour prédire correctement la SMO des arbustes fourragers (figure 1).

\section{Prédiction de la dMOgt}

Afin de faciliter la comparaison entre les résultats obtenus dans cette étude et ceux d'Arbelot (2), ses équations de prédiction de la dMOgt sont également présentées dans le tableau II. Cette comparaison scra analysće dans la partie Discussion de cet article. 


\section{TABLEAU}

Prédiction de la dégradabilité enzymatique de la matière organique (SMO - p. 100 MS) en fonction de critères chimiques pour quelques familles de fourrages ligneux
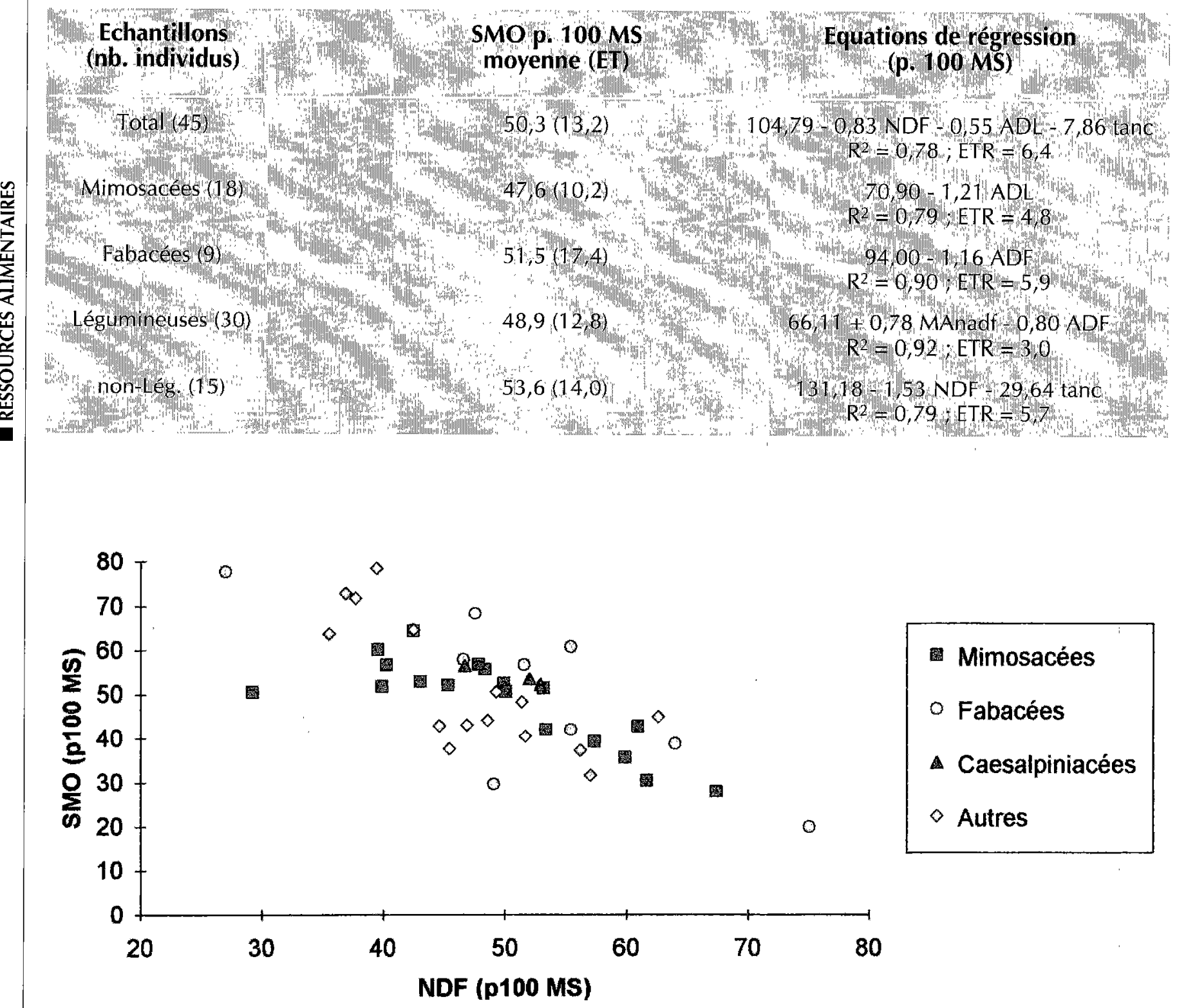

Mimosacées

- Fabacées

$\triangle$ Caesalpiniacées

$\diamond$ Autres

Figure 1 : solubilité de la matière organique (SMO) des ligneux fourragers en fonction de leur teneur en NDF.

Lorsque les critères chimiques sont seuls pris en compte, la qualité de la régression est médiocre $\left(\mathrm{R}^{2}<0,7\right)$. Exemple : équation de prédiction de la dMOgt en fonction des critères chimiques pour l'ensemble des ligneux fourragers (45):

dMOgt (p. $100 \mathrm{MO})=88,70-0,60 \mathrm{NDF}-0,17 \mathrm{ADL} / \mathrm{ADF}-2,38$ $\operatorname{tanpt}\left(\mathrm{R}^{2}=0,61 ; \mathrm{ETR}=4,8\right)$.

Aussi, afin d'améliorer sensiblement cette prédiction, la variable explicative SMO (critère enzymatique) est prise systématiquement en compte dans les équations présentées dans le tableau II.

Les équations de prédiction de la dégradabilité in vitro de la matière organique mesurée par le gastest de Hohenheim est relativement fiable pour les Fabacées $(\mathrm{CVr}=5,1$ p. 100) mais est médiocre pour les Mimosacées $\left(\mathrm{CVr}=9,6\right.$ p. 100 et $\left.\mathrm{R}^{2}=0,68\right)$.
Cela s'explique notamment par une plus grande dispersion des espèces de la famille des Fabacées par rapport aux autres familles (figure 2). Ceci se vérifiera d'ailleurs pour les autres critères.

\section{Prédiction des MApro (tableau III)}

Les équations de prédiction des matières azotées dégradées par la pronase (après une heure d'incubation) sont médiocres sauf pour les non-Légumineuses et les Fabacées bien que leurs coefficients de variations résiduels respectifs soient élevés (22 p. 100 ct 28 p. 100) (figure 3). La prédiction est particulièrement mauvaise pour les Mimosacées $\left(\mathrm{R}^{2}=0,28 ; \mathrm{CVr}=30\right.$ p. 100) et n'est donc pas applicable en pratique. MAnadf est néanmoins le meilleur critère de prédiction de MApro. 
TABLEAU II

Prédiction de la dégradabilité in vitro de la matière organique par le gastest (dMOgt - p. 100 MO) en fonction de critères chimiques et enzymatiques pour quelques familles de fourrages ligneux

Echantillons

(nb. individus)
dMOgt p. 100 MO

moyenne (ET)

\section{Equations de régression (p. $100 \mathrm{MO}$ )}

Corniaux et coll. (1996) : échantillons de feuilles, fleurs et fruits de ligneux fourragers

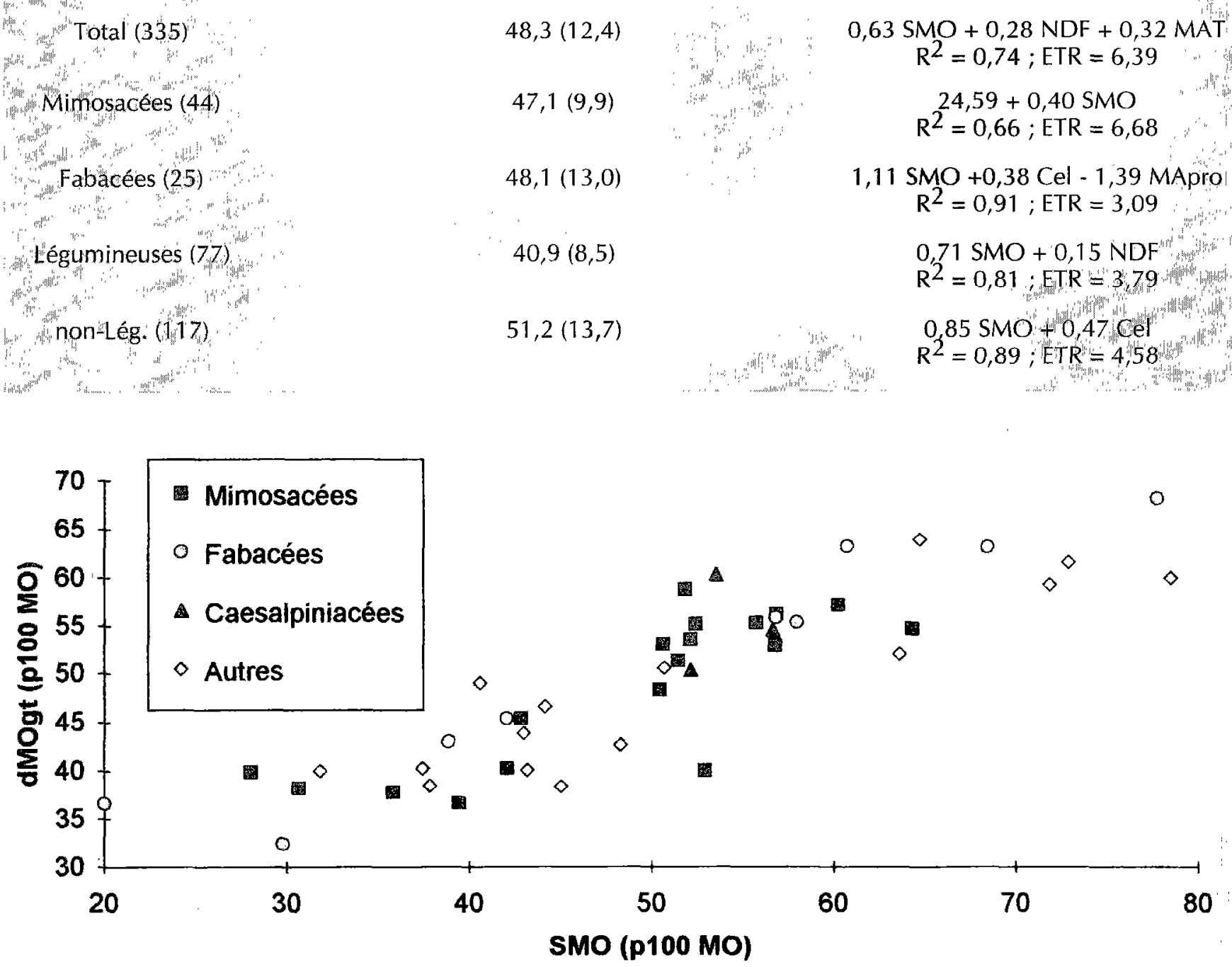

Figure 2 : dégradabilité par le gastest de la matière organique (dMOgt) des ligneux fourragers en fonction de la solubilité de leur matière organique $(S M O)$.

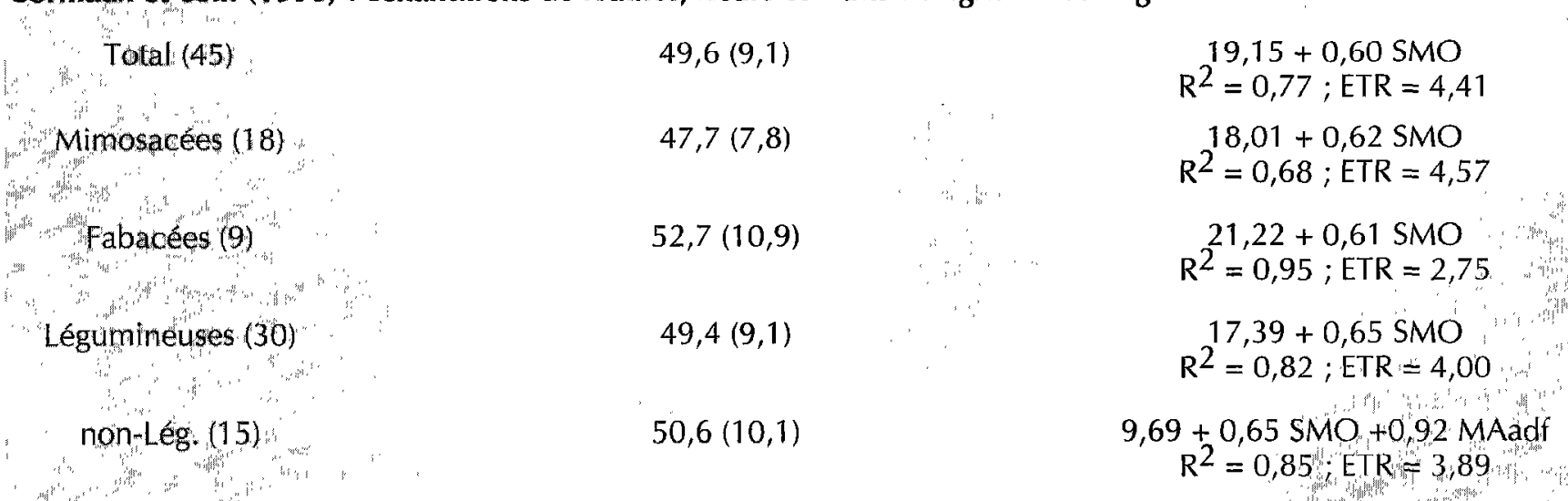

Arbelot (1993) : échantillons de feuilles de ligneux fourragers

$48,3(12,4)$ 
Prédiction des teneurs en matières azotées dégradées par la pronase en fonction de critères chimiques pour quelques familles de fourrages ligneux

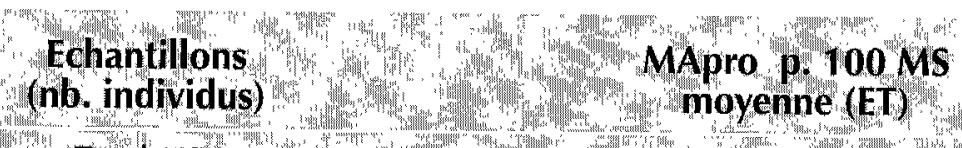

Equations de régression moyenne (ET) (p. 100 MS)

\section{Total (45)}

$4,3,2,6)$

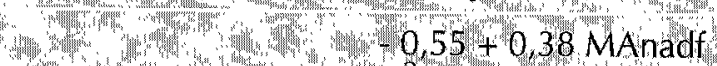

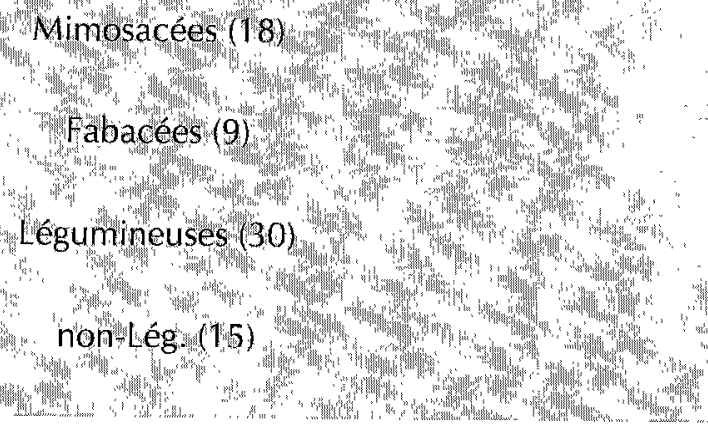

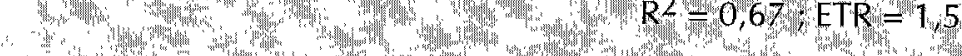

$4,4,(1,5)$

$111+24$ MAnadf

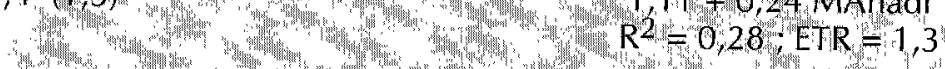

$6,5,(3,6)$

$4,31+0,5,2 \mathrm{MAnadf}$

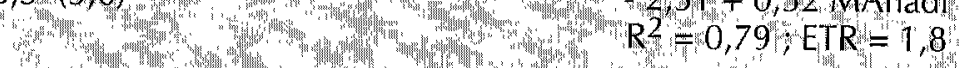

$5,1(2,6), 40,0,44$ Mnad

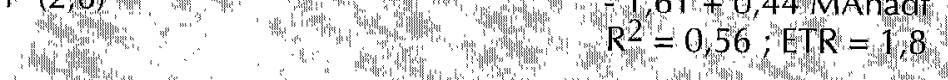

$2,7,(1,5)$

$011+0,32$ MAnadf

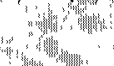

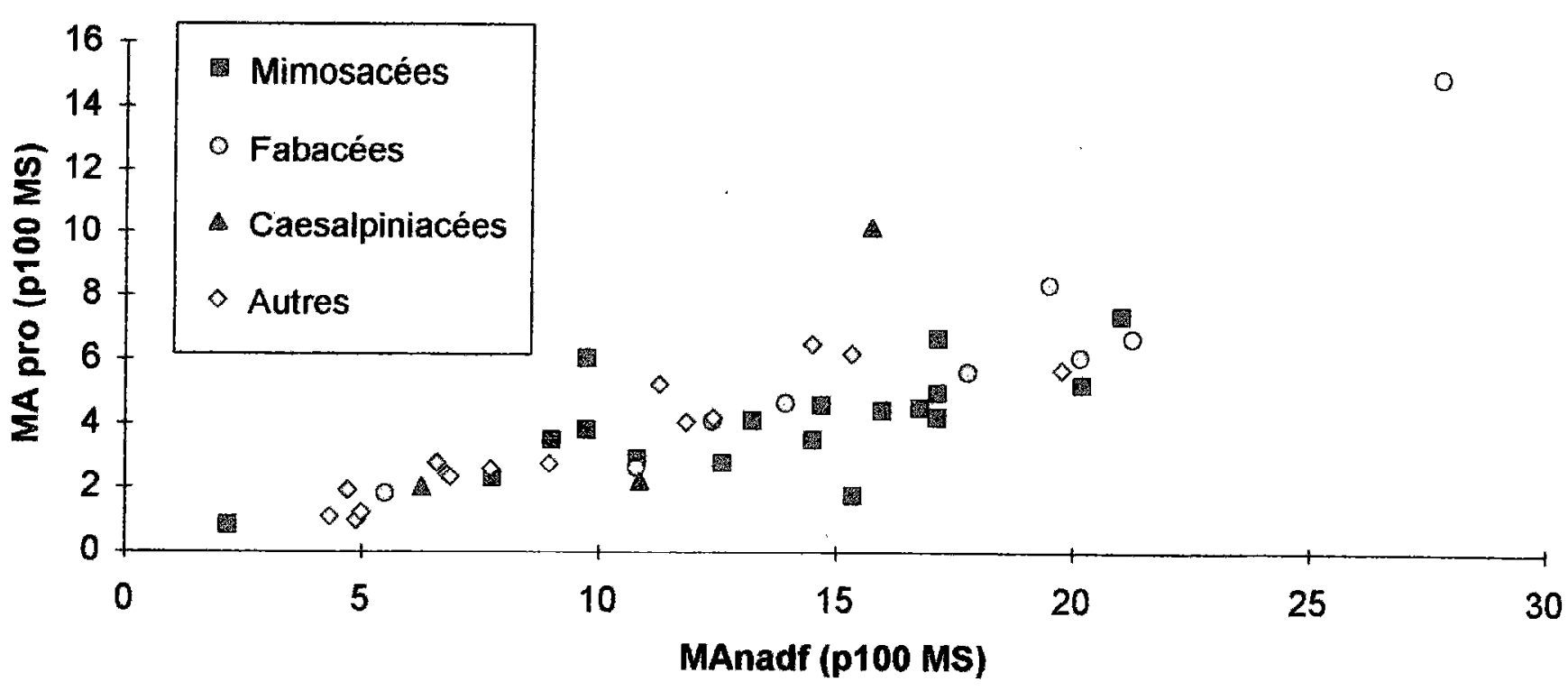

Figure 3 : matières azotées des ligneux fourragers dégradées par la pronase (MApro) en fonction do leur teneur en matières azotées non liées à I'ADF (MAnadf).

\section{Prédiction des MAgt}

Afin de faciliter la comparaison entre les résultats obtenus dans cette étude et ceux d'Arbelot (2), ses équations de prédiction de la MAgt sont également présentées dans le tableau IV. Cette comparaison sera analysée dans la partie Discussion de cet article.

Les équations de prédiction des matières azotées dégradées in vitro sont relativement médiocres avec notamment un coefficient de variation résiduel très élevé pour les Mimosacées $(\mathrm{CVr}=$ 59 p. 100). L'équation la plus précise est obtenue pour les nonLégumineuses $\left(\mathrm{R}^{2}=0,88 ; \mathrm{CVr}=20\right.$ p. 100).

\section{DISCUSSION}

Prédicteurs de la valeur nutritive des arbustes fourragers

Dans cette étude, trois types de critères ont été pris en considération : des critères chimiques, des critères enzymatiques et des critères in vitro. Plus proches des conditions physiologiques que les mesures de dégradabilitćs enzymatiques, les estimations de la digestibilité de la matière organique (dMOgt) et de la dégradabilité des matières azotées (MAgt) par le gastest sont probablement les 
TABLEAU IV

\section{Prédiction des teneurs en matières azotées dégradées in vitro par le gastest en fonction de critères chimiques pour quelques familles de fourrages ligneux}

Echantillons (nb, individus)
MAgt p. $100 \mathrm{MS}$ moyenne (ET)

\section{Equations de régression (p. $100 \mathrm{MS})$}

Corniaux et coll. (1996) : échantillons de feuilles, fleurs et fruits de ligneux fourragers

\begin{tabular}{|c|c|c|}
\hline Total $(45)$ & $3,79(3,2)$ & $\begin{array}{r}9,35+0,62 \text { MApro }-1,35 \text { tanc }-0,09 \text { ADF }-0,10 \text { ENA } \\
\text { R }^{2}=0,76 ; E T R=1,7\end{array}$ \\
\hline mosacées $(18)$ & $3,54 \quad(2,7)$ & $\begin{array}{l}-3,88+0,51 \text { MAnadf } \\
\mathrm{R}^{2}=0,44 ; E T R=2,09\end{array}$ \\
\hline Fabacées (9) & $6,61,(4,2)$ & $\begin{array}{l}-3,81+0,61 \text { MAnadf } \\
\mathrm{R}^{2}=0,81 ; \text { ETR }=1,99\end{array}$ \\
\hline Légumineuses $(30)$, & $4,58 \quad(3,6)$ & $\begin{array}{r}-3,83+0,37 \text { MAnadf }+0,55 \text { MApro } \\
\mathrm{R}^{2}=0,68 ; \text { ETR }=2,13\end{array}$ \\
\hline nón-Leg r $(15)$ & $2,21 \quad(1,1)$ & $\begin{array}{r}4,74-0,07 \text { ADF }-0,74 \text { tanpt }+0,08 \text { MAadf } \\
\left.R^{2}=0,88 ; E\right\rceil K=0,44\end{array}$ \\
\hline belot (1993) : échantillons & de ligneux & S $\quad \therefore$, n \\
\hline Total $(84)$ & $2,4(3,3)$ & $\begin{array}{c}-1,99+0,04 \text { SMS + 0,02 MAnadf }-0,24 \text { tanpt } \\
R^{2}=0,51 ; E T R=1,8\end{array}$ \\
\hline Mimosacées (18) & $2,7 \quad(3,0)$ & $\begin{array}{l}0,74 \text { MApro }+0,45 \text { MGE } \\
\mathrm{R}^{2}=0,58 ; \mathrm{ETR}=2,1\end{array}$ \\
\hline Fabacees $(11)$ & $3,1 \quad(4,1)$ & $\begin{array}{l}-17,34+0,30 \text { SMS }+1,50 \text { MApro } \\
\mathrm{R}^{2}=0,92 ; \mathrm{ETR}=0,8\end{array}$ \\
\hline $\begin{array}{l}\text { Légumineuses (41) } \\
\text { non-Lég. (43) }\end{array}$ & $2,4 \quad(3,1)$ & 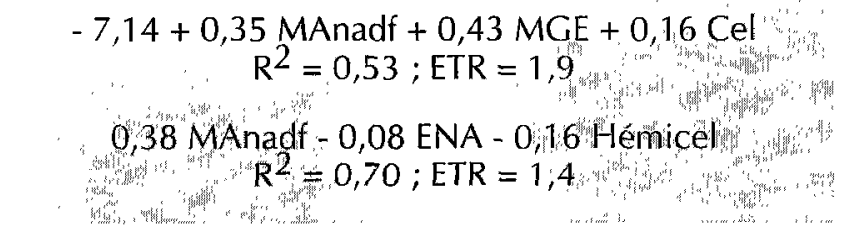 \\
\hline
\end{tabular}

plus fiables (2), bien que certaines études soient plus pessimistes à ce sujet (3). Par ailleurs, les critères chimiques sont les plus communément mesurés en laboratoire alors qu'il est actuellement difficile de mettre en ouvre de façon systématique les mesures in vitro. Aussi les critères enzymatiques (SMO, MApro) ont-ils été prédits à partir des critères chimiques et les critères in vitro (dMOgt, MAgt) à partir des critères chimiques seuls puis à partir des critères chimiques et enzymatiques.

Il apparait tout d'abord que les critères enzymatiques sont prédits de façon relativement médiocre à partir des critères chimiques avec toutefois de meilleurs résultats dans le cas des équations de régression concernant la SMO $\left(0,78<\mathrm{R}^{2}<0,90\right.$ et 6,1 p. $100<\mathrm{CVr}<12,7$ p. 100). Il en est de même pour la prédiction des critères in vitro à partir des critères chimiques seuls. En revanche, hormis pour les Mimosacées $\left(\mathrm{R}^{2}=0,68 ; \mathrm{CVr}=\right.$ 9,6 p. 100), la prédiction de la dMOgt est sensiblement améliorée lorsque la SMO, critère enzymatique, est prise en compte $(5,1$ p. $100<\mathrm{CVr}<8,9$ p. 100$)$.

Par ailleurs et en règle générale, les critères énergétiques (SMO et dMOgt) et azotés (MApro et MAgt) de la valeur nutritive des arbustes fourragers sont prédits préférentiellement à partir des te- neurs en ADF ou ADL (parois végétales) et à partir des tencurs en MAadf ou MAnadf (fractions azotées liées, ou non, aux parois). En cela, les ligneux fourragers se distinguent des espèces herbacées pour lesquelles les critères analytiques les plus simples (MAT, NDF) sont le plus souvent suffisants $(8,13)$. Ce résultat est par ailleurs conforme aux études précédentes $(2,10)$ qui confirment le rôle essentiel de la lignification des parois végétales, ainsi que la répartition de l'azote dans les tissus et les cellules, sur la valeur nutritive des arbustes.

Par contre, le rôle des tanins est plus difficile à cerner. La mesure des tanins condensés ( $\operatorname{tanc}$ ) semble plus intéressante que celle des tanins précipitants $(\tan p t)$ qui ne figurent que dans une seule équation. Cependant, la concentration relative des tanins solubles et insolubles (condensés) est variable au sein même d'une espèce en fonction de la saison, du site ou de l'organe, ce qui accentue la difficulté de prédire la valeur nutritive des fourrages ligneux sur la base de leur analyse chimique (11).

\section{Prédiction par famille ou par groupe végétal}

Les équations de régression établies par groupe végétal sont plus précises pour les non-Légumineuses comparativement aux 
Légumineuses. Il est d'ailleurs notable que les variables indépendantes utilisées sont différentes entre les deux groupes. En particnlier, les tanins apparaissent dans les équations de prédiction de MAgt et de SMO pour les non-Légumineuses. Ceci tend à confirmer le rôle plus important de ces facteurs antinutritionnels dans la valeur nutritive des espèces non-Légumineuses comparées aux Légumineuses. Une différence de structure chimique de ces composés entre les deux groupes pourrait expliquer ce résultat $(2,15)$.

Les équations de régression établies par famille montrent une meilleure prédiction de tous les critères pour les Fabacées par rapport aux Mimosacées. Ceci est conforme aux études précédentes (2). Pour les Fabacées, la plus grande dispersion de ces critères (ET Fabacées > ET Mimosacées) permet d'améliorer sensiblement la validité des équations (figure 1). Pour les Mimosacées, l'hétérogénéité chimique interspécifique élevée pour les matières azotées non liées à l'ADF et surtout pour les tanins précipitants (coefficient de variation respectif de 25 p. 100 et de 181 p. 100), peut expliquer les difficultés à préciser ce modèle de prédiction $(8,12)$.

\section{Cas particuliers de dMOgt et de MAgt}

Comparées aux équations de prédiction de dMOgt établies par Arbelot (1993), les équations présentées dans cette étude sont globalement plus précises. La moyenne des $\mathrm{R}^{2}$ est en effet respectivement pour les deux études de 0,80 et 0,82 , celle des ETR étant de 4,9 et 3,9 (tableau II). Cependant, comme cela a été précisé auparavant, la hiérarchie entre les groupes botaniques n'est pas modifiée. Le principal critère de prédiction est également le même (SMO).

En ce qui concerne la prédiction de MAgt, les équations sont relativement médiocres dans les deux études $\left(\mathrm{R}^{2}<0,7\right)$, sauf pour les Fabacées et les non-Légumineuses (tableau IV). La hiérarchie entre les groupes botaniques reste globalement inchangée. En revanche, les facteurs de prédiction diffèrent, même si MAnadf apparaît régulièrement dans les équations.

La différence de précision des équations entre les deux études, bien que faible, peut s'expliquer par le nombre d'échantillons sensiblement plus élevé dans l'étude d'Arbelot. Pour les ligneux fourragers, l'hétérogénéité de composition chimique des groupes botaniques, et notamment de la famille des Mimosacées, rend en effet difficile l'application d'une équation générale de prédiction. Il est donc préférable de s'intéresser à l'espèce ou, dans certains cas, à la famille (Fabacées) (2).

Il faut également noter que, dans cette étude, des échantillons (8 sur 45) ont été récoltés avec fleurs ou fruits alors que le travail d'Arbelot ne considérait que les feuilles. Il s'agit d'un biais certain qui mérite d'être quantifié.

\section{Limites et prolongements de l'étude}

Même si le nombre de ligneux fourragers concernés est faible (8 sur 45), le mélange feuilles, tiges, fleurs et fruits est défavorable à la précision de ces équations de prédiction. Un travail complémentaire par organe prélevé pourrait affiner cette analyse.

Par ailleurs, plusieurs études $(14,18)$ ont montré que des facteurs géographiques, climatiques, édaphiques et phénologiques pouvaient être responsables de variations intraspécifiques. Or ces échantillons, prélevés à la même période mais à des stades phénologiques différents, proviennent de localisations très diverses sur le plan pédo-climatique (côte Ouest, côte Est, Sud de la NouvelleCalédonie, îles Loyauté). Comme pour le point précédent, une étude complémentaire qui incrémenterait notre base de données, permettrait de fiabiliser ce modèle de prédiction.
Enfin, et il s'agit probablement de la limite essentielle de cette étude, il faut rester prudent quant à la qualité de prédiction de dMOgt. En effet, faute de mesure de la dMO in vivo, il n'a pas été possible aux auteurs d'établir une hiérarchie entre les différentes méthodes de prédiction (dégradabilité de la matière organique par la pepsine-cellulase (4), digestibilité in vitro de Tilley et Terry (22), dMOgt (17)). L'hypothèse de travail retenue ici, qui considère la dMOgt plus fiable que la SMO, devra donc être vérifiée à l'avenir. Sous cette condition, les équations de prédiction de la dMOgt permettront d'évaluer convenablement la digestibilité de la matière organique dans de nombreux laboratoires qui ne disposent pas des moyens de mesure les plus performants.

\section{CONCLUSION}

Cette étude a permis d'établir des équations de prédiction de quelques facteurs liés à la valeur nutritive des ligneux fourragers tropicaux. Elle montre notamment la difficulté de déterminer ces équations à partir de critères chimiques simples (MAT, NDF) couramment utilisés pour les espèces herbacées. Leur teneur généralement élevée en lignines, la distribution de leurs matières azotées partiellement bloquées au niveau des parois indigestibles et la présence de tanins rendent nécessaire l'emploi de critères chimiques tels qu'ADL, MAadf et MAnadf dans le cas des arbres et arbustes fourragers. Des études complémentaires sur la nature des lignines et des tanins permettront sûrement d'affiner ces modèles de prédiction.

La précision des équations est néanmoins acceptable notamment pour la dMOgt et pour la famille des Fabacées. En revanche, les équations ne sont pas applicables pour MApro (sauf pour les Fabacées).

Mais cette étude n'est qu'une étape dans le programme de recherches des auteurs, l'objectif final étant l'implantation chez les éleveurs d'espèces arbustives adaptées à leurs conditions pédo-climatiques, valorisées par les ruminants et permettant le maintien de la fertilité des sols. Aussi des travaux complémentaires sont-ils actuellement menés en Nouvelle-Calédonie pour déterminer la productivité, le pouvoir de fixation de l'azote et l'appétabilité de ligneux arbustifs. Des essais de gestion de cette ressource fourragère sont également à l'étude.

\section{Remerciements}

Ce travail a été réalisé dans le cadre du programme de la CEEDGXII. Aussi, nous tenons à adresser nos sincères remerciements aux trois laboratoires (Laboratoire d'analyse des aliments du bétail, Nouméa; Laboratoire d'alimentation CIRAD-EMVT de Maisons-Alfort; Institut für Tierernährung, Universität Hohenheim) qui nous ont aidés dans cette étude.

\section{BIBLIOGRAPHIE}

1. AFNOR, 1980. Recueil de normes françaises des méthodes générales d'analyse des produits agro-alimentaires. Chimie - microbiologie - analyse sensorielle. Paris, France, AFNOR, 278 p.

2. ARBELOT $B:$ 1993. Prédiction de la valeur nutritive des fourrages ligneux tropicaux. Maisons-Alfort, France, CIRAD-EMVT. (Rapport de stage).

3. AUFRERE 3., GUERIN H., 1996. Critical review of chemical and enzymatic methods for the estimation of nutritive value in roughages. $A n n$. Zootechn, 45 suppl. : 11-23. 
4. AUfRERE J., MICHALET-DOREAU B., 1990. Nouvelles méthodes d'estimation de la valeur alimentaire des fourrages. II. Méthodes enzymatiques. Fourrages, $122:$ 203-217.

5. BIPEA, 1976-81. Recueil des méthodes d'analyse des Communautés européennes. Paris, France, ITCF.

6. CARTAILLER D., 1988. Mise au point d'une méthode de prévision de la dégradabilité des protéines alimentaires des aliments concentrés dans le rumen. Ann. Zootech., $37: 255-270$.

7. CIRAD-EMVT, 1993. Caractéristiques et valeurs alimentaires des fourrages en Nouvelle-Calédonie. Nouméa, Nouvelle-Calédonie, CIRADEMVT, $150 \mathrm{p}$.

8. CORNIAUX C., 1991. Recherche sur Maré des espèces ayant un intérêt fourrager. Maisons-Alfort, France, CIRAD-EMVT, 11 p. (Etudes et synthèses)

9. CORNIAUX C., DURAND N., SARRAIHL J.M., GUERIN H., 1996. Teneurs en éléments bruts et dégradabilité enzymatique d'espèces ligneuses arbustives utilisables par les ruminants dans les parcours extensifs de Nouvelle-Calédonie. I. Typologie. Revue Elev. Méd. vét. Pays trop., $49: 60-67$.

10. DURAND N., 1993. Identification d'espèces ligneuses arbustives utilisables par les ruminants dans les parcours extensifs en NouvelleCalédonie. Nouméa, Nouvelle-Calédonie, CIRAD-Elevage, 54 p. (Etudes et synthèses)

11. GRILLET C., VILLENEUVE F., 1994. Les tanins dans les fourrages ligneux. Chapitre VI. In : Guerin H. éd. Valeur alimentaire des fourrages ligneux consommés par les ruminants en Afrique centrale et de l'Ouest. Commission des Communautés européennes, DGXII, programme ST2.A/89/215.F, Maisons-Alfort, France, CIRAD-EMVT, 393 p.

12. GUERIN H., 1994. Echantillonnage des fourrages ligneux, analysés au laboratoire, Composition chimique et dégradabilité enzymatique. Chapitre V. In : Guerin H. éd., Valeur alimentaire des fourrages ligneux consommés par les ruminants en Afrique centrale et de l'Ouest. Commission des Communautés européennes, DGXII, programme ST2.A/89/215.F, Maisons-Alfort, France, CIRAD-EMVT, 393 p.
13. GUERIN H., RICHARD D., LEFEVRE P., FRIOT D., MBAYE N:, 1989. Prévision de la valeur nutritive des fourrages ingérés sur parcours naturels par les ruminants domestiques sahéliens et soudaniens. In : XVI Congrès international des herbages, Nice, France, 4-11 octobre 1989. Versailles, France, AFPF, INRA, p. 879-880.

14. ICKOWICZ A., DASSERING O., 1994. Extrait de: Echantillonnage des fourrages ligneux - $A$ nalyses au laboratoire Composition chimique et dégradabilité enzymatique. In : Guerin H. éd. Valeur alimentaire des fourrages ligneux consommés par les ruminants en Afrique centrale et de I'Ouest. Commission des Communautés européennes, DGXII, programme ST2.A/89/215.F, Maisons-Alfort, France, CIRAD-EMVT, 393 p.

15. LEINMULLER E., STEINGASS H., MENKE K.H., 1991. Tanins in ruminants feedstuffs. Anim. Res. Dev., 33: 62 p.

16. MASON V.L., 1969. Some observations on the distribution and origin of nitrogen in sheep faeces. J. Agric. Sci., Camb., 73: 99-111.

17. MENKE K.H., STEINGASS H., 1988. Estimation of the energic feed value obtained from chemical analysis and in vitro gas production using rumen fluid. Anim. Res. Dev., 28: 7-55.

18. NORTON B.W., 1994. The nutritive value of tree legumes. In: Forage tree legumes in tropical agriculture. Wallingford, United Kingdom, CAB International, $389 \mathrm{p}$.

19. SCALBERT A., MONTIES B., JANIN G., 1987. Comparaison de méthodes de dosage des tanins : application à des bois de différentes espc̀ccs. $2^{\mathrm{e}}$ colloque Sciences et industries du bois, tome II, thèmes 3,4 et 5, Nancy, France, 22-24 avril 1987, Nancy, France, A.R.Bo.Lor.

20. SHELTON H.M., BREWBAKER J.L., 1994. Leucaena leucocephalathe most widely used forage tree legume. In: Forage tree legumes in tropical agriculture. Wallingford, United Kingdom, CAB International, $389 \mathrm{p}$.

21. SPSS, 1992. Base system user's guide. Version 5.0., $910 \mathrm{p}$.

22. TILIEY J.M., TERRY R.A., 1963. A two stage technique for in vitro digestion of forage. J. Br. Gram. Soc., 18: 104-111.

23. VAN SOEST P.J., WINE R.H., 1967. Use of the detergents in the analysis of fibrous feeds. I. Ass. Off. Anal., 50: 50-55.

Reçu le 2.5.95, accepté le 11.9 .96 


\section{Summary}

Corniaux C., Guerin H., Steingass H. Chemical components and enzyme and in vitro digestibility of woody shrubs used by ruminants in New Caledonian rangelands. II. Prediction equations of enzyme and in vitro digestibility of crude protein and organic matter

In New Caledonia, as in many other countries, it is not realistic to consider in the short-term using all fodder assessment methods. Compare to herbaceous fodder, tropical shrubs nutritive value is not well known. Thus, prediction equations of some factors associated with the nutritive value of tropical shrubs and trees collected in New Caledonian extensive lands were computed in this study. On one hand, criteria were determined by enzyme methods: organic matter enzyme digestibility (SMO) and crude protein digestibility by pronase (MApro); on the other hand, by the in vitro method: in vitro digestibility of organic matter (dMOgt) and crude protein (MAgt) via the gas production method. This study shows the difficulty to provide prediction equations using chemical compound contents, which are commonly used for herbaceous species (MAT, NDF, ADF). Because of their usually high lignin content, their crude proteins partly bound to undigestible fibers and their tannin content, the use of chemical criteria such as ADL, MAadf and MAnadf (MAT bound or not to ADF) is necessary for fodder shrubs and trees. The accuracy of the equations is nevertheless reasonable for dMOgt and for the Fabacae family. On the other hand, equations based on MApro are not satisfactory except for Fabacae.

Key words: Woody plant - Nutritive value - Statistical analysis - Chemical composition - Digestibility - Organic matter Digestible nitrogen - Ruminant - Rangeland - New Caledonia.

\section{Resumen}

Corniaux C., Guerin H., Steingass H. Composición química y degradación enzimática e in vitro de las especies de arbustos leñosos utilizables por los rumiantes en los recorridos extensivos en Nueva Caledonia. II. Ecuaciones de predicción de la degradación enzimática e in vitro de la materia orgánica y de las materias nitrogenadas

En Nueva Caledonia, como en muchos otros países, es imposible utilizar a corto plazo todos los métodos de evaluación de los forrages. Por otru lado, el valur alimenticio de los arbustos forrageros es relativamente desconocido, en comparación con el de las herbáceas. Fl presente estudio establece las ecuaciones de predicción de algunos factores ligados al valor nutritivo de los leñosos tropicales recolectados en las zonas de los recorridos neo caledónicos. Se trata por un lado de criterios determinados por los métodos enzimáticos : degradación enzimálica de la materia orgánica (SMO) y de las materias nitrogenadas degradadas por la pronasa (MApro) ; y por otro lado, por el método in vitro : degradación in vitro de la materia orgánica (dMOgt) y de las materias nitrogenadas (MAgt) mediante el método de producción de gaz. Este estudio muestra principalmente la dificultad para determinar estas ecuaciones a partir de criterios químicos simples (MAT, NDF, ADF), utilizados frecuentemente para las especies herbáceas. La tenencia en lignina, generalmente elevada, la distribución de las materias nitrogenadas parcialmente bloqueadas a nivel de las paredes indigeribles y la presencia de taninos, obligan al uso de criterios químicos como el ADL, MAadf y MAnadf (materias nitrogenadas ligadas o no al ADF) en el caso de los árboles y arbustos forrageros. Sin embargo, la precisión de las ecuaciones es aceptable, sobre todo para la dMOgt y para la familia de las Fabáceas. Por el contrario, las ecuaciones no son aplicables para la MApro (excepto para las Fabáceas).

Palabras clave : Planta leñosa - Valor proteínico - Análisis estadístico - Composición química - digestibilidad - Materia orgánica - Nitrogeno asimilable - Rumiante - Tierra de pastos - Nueva Caledonia. 